\title{
A NOTE ON WITHDRAWAL FROM A FLUID OF FINITE DEPTH THROUGH A POINT SINK
}

\author{
G. C. HOCKING ${ }^{1}$, J.-M. VANDEN-BROECK ${ }^{2}$ and L. K. FORBES ${ }^{3}$
}

(Received 21 September, 1998; revised 3 September, 1999)

\begin{abstract}
The problem of withdrawal through a point sink of water from a fluid of finite depth with a free surface is considered. Assuming the flow to be axisymmetric, it is found that there is a maximum Froude number at which such flows can exist. This maximum corresponds to the formation of a secondary stagnation ring on the free surface. This result extends earlier work on this problem. Comparison is made with a small Froude number solution and past experimental results.
\end{abstract}

\section{Introduction}

The withdrawal of water from a single fluid with a free surface or two layers of different density is important in a number of applications. These include rapid withdrawal from fuel tanks in aircraft or spacecraft and water holding tanks in fire fighting vehicles. The process of withdrawal of water from storage reservoirs, solar ponds and cooling ponds is vital in maintaining water quality and efficient operation [10]. An understanding of the withdrawal process is important to enable us to model these situations and improve management strategies.

Experiments $[7,8,11-13,18]$ show that when withdrawal occurs through a circular orifice at the bottom of a fluid consisting of two layers of different density (which may be air and water), buoyancy forces ensure that only fluid from the lower layer is drawn through the hole at low values of the flow rate. As the depth of the lower layer decreases the effective flow rate increases, and at some critical value the interface is pulled down so that the upper layer also flows out. This critical transition from one to two layers occurs in a matter of seconds.

It is of interest to examine this flow in a simplified framework to determine the mechanisms which lead to this critical transition. If the effective flow rate is below the

\footnotetext{
'Mathematics and Statistics, DSE, Murdoch University, Murdoch, WA, 6150, Australia.

${ }^{2}$ Department of Mathematics, University of East Anglia, Norwich, UK.

${ }^{3}$ School of Mathematics and Physics, The University of Tasmania, Hobart, TAS 7001, Australia.

(C) Australian Mathematical Society 2002, Serial-fee code 1446-1811/02
} 
critical value, the flow can be modeled as a single-layer flow with gravity $g$ replaced by the effective gravity $g^{\prime}=(\Delta \rho / \rho) g$, where $\Delta \rho$ is the difference in density between the two layers and $\rho$ is some reference density. In this simplified model the fluid is assumed to be inviscid and incompressible and the flow to be steady and irrotational.

The flow can be shown to be characterised by the Froude number, $F=\left(m^{2} / g^{\prime} H^{5}\right)^{1 / 2}$, where $m$ is the total flux from the point sink and $H$ is a length scale. The Froude number can be thought of as the effective flow rate, so an increase in $F$ means an increase in flux out through the point sink. We will denote by $F_{C}$ the critical Froude number at which the interface (between two fluids of different density) or free surface is drawn down into the sink.

If the fluid is of infinite depth, Forbes and Hocking [4] and Vanden-Broeck and Keller [17] have shown that for small values of the Froude number there is a stagnation point directly above the point sink on the interface. As the flow rate increases the flow breaks down when a stagnation ring forms on the free surface a short distance from the central stagnation point. Vanden-Broeck and Keller [17] examined the nature of this stagnation ring.

In the case of a finite depth fluid, Forbes et al. [6] used a distributed singularity method to obtain solutions for a Froude number up to a value of $F=1.5$, but were unable to obtain solutions at higher values. No stagnation ring was observed to form on the free surface and no reason was found to explain the breakdown of the solutions. In this paper a modification of the numerical scheme used by Forbes and Hocking [4] is used to extend solutions to higher values of $F$, and it is found that the stagnation ring forms in this case also, as in the case of axisymmetric flow from a fluid of infinite depth.

There exists an analogous problem of withdrawal through a line sink, and there is a much greater amount of literature on this problem, see for example $[3,9,14-16]$. In this two dimensional problem, solutions both with a stagnation point on the surface and others with a downward cusp above the sink have been obtained. In the two dimensional case, Hocking [9] showed that these cusp solutions appear to be the event which precedes the critical transition to two layer flow. No cusped solutions have ever been found in the problem of axisymmetric flow into a point sink.

In Section 2, the model is formulated, and in Section 3 the first term of a small Froude number expansion is obtained. Section 4 describes the numerical scheme that is used to solve this system, and Section 5 discusses the results in the context of other work on this problem.

\section{Problem formulation}

The steady, axisymmetric, irrotational motion of an inviscid, incompressible fluid of finite depth in three dimensions is to be examined. The fluid lies beneath a free 
surface and is being drawn out through a point sink situated on the bottom of the fluid at a depth $H$. Figure 1 shows the configuration of the flow domain.

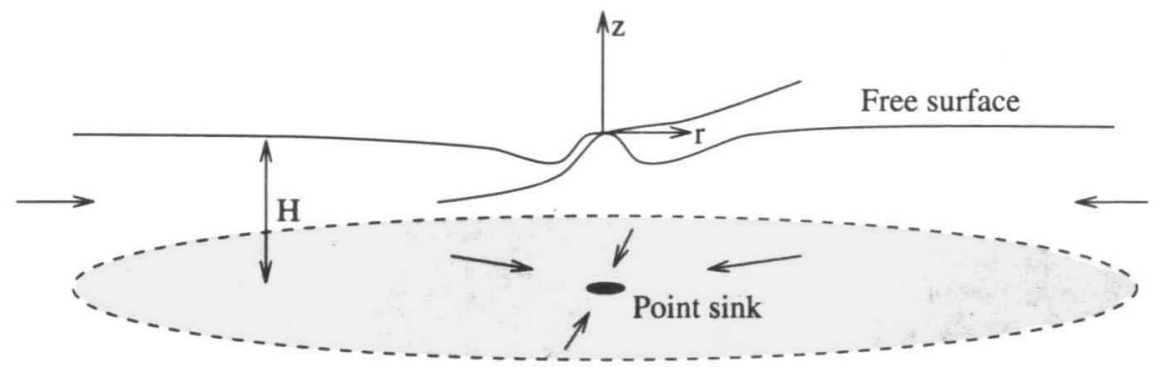

FIGURE 1. Figure defining the problem under consideration.

In cylindrical polar $(r, \theta, z)$ coordinates this becomes a two dimensional problem in $(r, z)$ if we assume the flow to be axisymmetric, and there exists a velocity potential $\Phi$ such that

$$
\nabla^{2} \Phi(r, z)=\Phi_{r r}+\frac{\Phi_{r}}{r}+\Phi_{z z}=0
$$

where the origin of the coordinate system is situated on the free surface directly above the sink, which is on the bottom at $(r, z)=(0,-H)$. As the sink is approached, this velocity potential must have the correct behaviour, which is

$$
\Phi \rightarrow \frac{m}{4 \pi \sqrt{r^{2}+(z+H)^{2}}} \quad \text { as }(r, z) \rightarrow(0,-H)
$$

where $m$ is the total flux from the region.

There can be no flow through the solid bottom boundary, so that

$$
\nabla \Phi \cdot \mathbf{k}=0, \quad z=-H
$$

On the free surface (or interface), a kinematic condition

$$
\Phi_{z}=\Phi_{r} \zeta_{r}, \quad \text { on } z=\zeta(r)
$$

must be satisfied. In addition a dynamic condition which ensures that pressure is constant along the free surface must be applied. If we non-dimensionalise with respect to the velocity $m / H^{2}$ and the length $H$, this condition can be written as

$$
\hat{\zeta}(\hat{r})+\frac{1}{2} F^{2}\left(\hat{\Phi}_{r}^{2}+\hat{\Phi}_{z}^{2}\right)=0, \quad \text { on } \hat{z}=\hat{\zeta}(\hat{r}),
$$




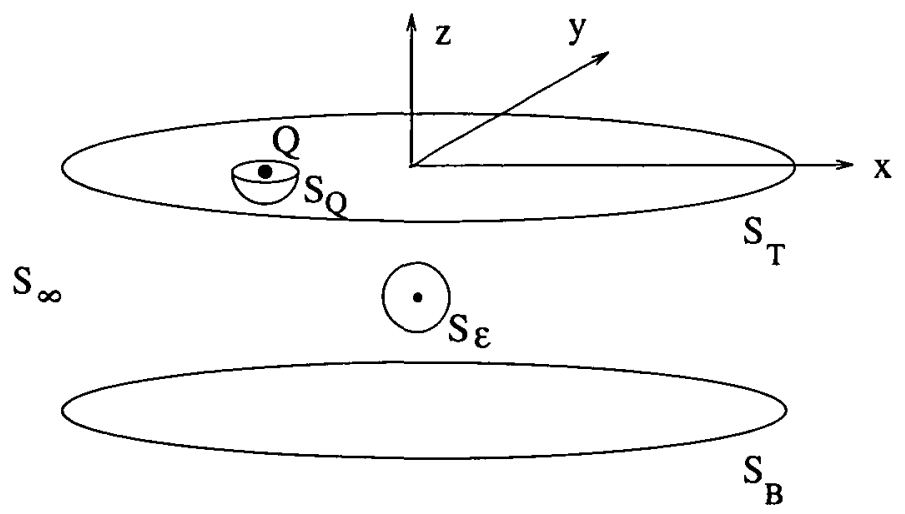

FIGURE 2. Surfaces used in derivation of the integral equation (2.6).

where $F=\sqrt{m^{2} / g H^{5}}$ is the Froude number. The form of the other equations is unchanged if the dimensional variables are replaced by their nondimensional equivalents. Henceforth we will drop the hat for convenience and work in nondimensional variables.

In order to formulate a mathematical problem to solve for this flow, we modify the derivation used in Forbes and Hocking [4], and use Green's second identity to derive an integral equation for the unknown harmonic function $\Phi(r, z)$. An image-free surface must be placed on the other side of the line $z=-1$, so that by symmetry the combination satisfies the condition of no flow normal to the bottom of the region (2.2).

Let point $Q$ be a fixed point on the free surface with coordinates $(r, \theta, \zeta(r))$ in cylindrical polar coordinates. Let $P(\alpha, \beta, \mu)$ be another point which is free to move over the same surface. Following the derivation in [4], we note that $\Phi$ is an harmonic function over the full region except at the sink itself, and then define another function,

$$
\Psi=\frac{1}{R_{P Q}}=\frac{1}{\left[r^{2}+\alpha^{2}-2 r \alpha \cos (\beta-\theta)+(z-\mu)^{2}\right]^{1 / 2}},
$$

which is also analytic except when $P$ and $Q$ are the same point.

Invoking Green's identity over the surfaces indicated in Figure 2, including the free surface and its image, a hemisphere omitting the point $Q$, a sphere omitting the sink and a cylindrical surface as $r \rightarrow \infty$, we obtain an integral equation of the form

$$
\begin{aligned}
2 \pi \Phi(Q)= & \frac{2}{\left(r^{2}+(z+1)^{2}\right)^{1 / 2}}-\iint_{S_{r}} \Phi(P) \frac{\partial}{\partial n_{P}}\left(\frac{1}{R_{P Q}}\right) d S_{P} \\
& -\iint_{S_{B}} \Phi(P) \frac{\partial}{\partial n_{P}}\left(\frac{1}{R_{P Q}}\right) d S_{P},
\end{aligned}
$$

where $S_{T}$ is the free surface and $S_{B}$ is its image. We know that symmetry provides 
a direct relationship between the variables on these two surfaces and we later exploit this fact to allow us to integrate only over the upper surface.

It is very important to notice that (2.6a) can also be written in the forms

$$
\begin{aligned}
2 \pi \Phi(Q)= & \frac{2}{\left(r^{2}+(z+1)^{2}\right)^{1 / 2}}-\iint_{S_{T}}[\Phi(P)-\Phi(Q)] \frac{\partial}{\partial n_{P}}\left(\frac{1}{R_{P Q}}\right) d S_{P} \\
& -\iint_{S_{B}} \Phi(P) \frac{\partial}{\partial n_{P}}\left(\frac{1}{R_{P Q}}\right) d S_{P}
\end{aligned}
$$

and

$$
\begin{aligned}
0= & \frac{2}{\left(r^{2}+(z+1)^{2}\right)^{1 / 2}}-\iint_{S_{T}}[\Phi(P)-\Phi(Q)] \frac{\partial}{\partial n_{P}}\left(\frac{1}{R_{P Q}}\right) d S_{P} \\
& -\iint_{S_{B}}[\Phi(P)-\Phi(Q)] \frac{\partial}{\partial n_{P}}\left(\frac{1}{R_{P Q}}\right) d S_{P} .
\end{aligned}
$$

The reason for this is that

$$
\iint_{S_{T}} \frac{\partial}{\partial n_{P}}\left(\frac{1}{R_{P Q}}\right) d S_{P}=0 \text { and } \iint_{S_{B}} \frac{\partial}{\partial n_{P}}\left(\frac{1}{R_{P Q}}\right) d S_{P}=-2 \pi
$$

so that these two terms can be added or removed as we choose. That this is true can be shown by noting that the "flux" through any horizontal plane due to the "sink" term $1 / R_{P Q}$ is equal to $-2 \pi$. It turns out that this is very important in obtaining a numerical solution to this problem. In the form (2.6a) the point $P=Q$ is singular, and so integrating through it causes a significant loss of accuracy. On the other hand, the form (2.6c) turns out to be numerically unstable as $F$ increases, as discussed in Forbes et al. [6]. The form (2.6b), however, turns out to be both accurate and stable numerically, enabling us to compute solutions until a critical $F$ is reached.

Starting with (2.6b) and again following Forbes and Hocking [4], and also noting that on the image surface $\Phi(\alpha,-2+\zeta(\alpha))=\Phi(\alpha, \zeta(\alpha))$, we arrive at the integral equation

$$
\begin{aligned}
2 \pi \Phi(Q)= & \frac{2}{\left(r^{2}+(z+1)^{2}\right)^{1 / 2}}-\int_{0}^{\infty}(\Phi(P)-\Phi(Q)) \mathscr{K}(a, b, c, d) d \alpha \\
& -\int_{0}^{\infty} \Phi(P) \mathscr{K}(e, b, f, d) d \alpha,
\end{aligned}
$$

in which the kernel function was shown by Forbes and Hocking [4] to be of the form

$$
\mathscr{K}(a, b, c, d)=\frac{2}{\sqrt{c+d}}\left[\zeta_{\alpha} \mathbf{K}\left(\frac{2 d}{c+d}\right)+\frac{2 a r-\zeta_{\alpha} c}{c-d} \mathbf{E}\left(\frac{2 d}{c+d}\right)\right]
$$


where $\mathbf{K}$ and $\mathbf{E}$ are the complete elliptic integrals of the first and second kind as defined in Abramowitz and Stegun [1], and the functions $a$ to $f$ are defined as

$$
\begin{array}{lll}
a=\alpha \zeta_{\alpha}(P)-(\zeta(P)-\zeta(Q)), & b=r \zeta_{\alpha}(P) \\
c=\alpha^{2}+r^{2}+(\zeta(P)-\zeta(Q))^{2}, & & d=2 r \alpha, \\
e=\alpha \zeta_{\alpha}(P)-(2+\zeta(P)+\zeta(Q)), & f=\alpha^{2}+r^{2}+(2+\zeta(P)+\zeta(Q))^{2}
\end{array}
$$

It is convenient to solve this problem using a formulation based on arclength along the surface, so that $s$ is the distance from $\alpha=0$ to $Q$, and $\sigma$ is the distance along the surface to $P$. The standard formula

$$
\left(\frac{d r}{d s}\right)^{2}+\left(\frac{d \zeta}{d s}\right)^{2}=1
$$

defines the arclength $s$ in terms of $r$ and $\zeta$.

Rewriting the integral equation (2.7) in terms of arclength, we obtain

$$
\begin{aligned}
2 \pi \phi(s)= & \frac{2}{\left(r^{2}(s)+(\zeta(s)+1)^{2}\right)^{1 / 2}}-\int_{0}^{\infty}(\phi(\sigma)-\phi(s)) \mathscr{K}(A, B, C, D) d \sigma \\
& -\int_{0}^{\infty} \phi(\sigma) \mathscr{K}(E, B, F, D) d \sigma,
\end{aligned}
$$

where $\phi(s)$ is the potential along the free surface and

$$
\begin{array}{ll}
A=r(\sigma) \zeta^{\prime}(\sigma)-r^{\prime}(\sigma)(\zeta(\sigma)-\zeta(s)), & B=r(s) \zeta^{\prime}(\sigma) \\
C=r^{2}(\sigma)+r^{2}(s)+(\zeta(\sigma)-\zeta(s))^{2}, & D=2 r(s) r(\sigma) \\
E=r(\sigma) \zeta^{\prime}(\sigma)-r^{\prime}(\sigma)(2+\zeta(\sigma)+\zeta(s)), & F=r^{2}(\sigma)+r^{2}(s)+(2+\zeta(\sigma)+\zeta(s))^{2}
\end{array}
$$

Eliminating $\Phi_{z}$ from the Bernoulli equation (2.4) and the kinematic condition (2.3) and combining leads to a single relation,

$$
\frac{1}{2} F^{2}\left(\frac{d \phi}{d s}\right)^{2}+\zeta(s)=0,
$$

on the free surface $z=\zeta(r)$.

The integral equation (2.11) is coupled with the condition (2.12), subject to (2.10), to give the complete formulation of the problem. This form of the integral equation has a logarithmic singularity in the complete elliptic integral $\mathbf{K}$ in the integrand as $r \rightarrow \sigma$ in the first integral. Forbes and Hocking [4] removed this singularity to create a non-singular integral equation, but doing so in the finite depth problem produced a system which was numerically unstable and failed to converge, leading them to use a different method altogether [6]. We will return to this matter later. 


\section{A solution for small Froude number}

It is possible to obtain a solution valid for small Froude number by expanding about a flat free surface, $\zeta=0$. We only compute the first term of this solution, since to compute higher order terms leads to prohibitively difficult algebra for little gain, since we will later solve the problem numerically.

Let

$$
\Phi(r, z)=\Phi_{0}(r, z)+F^{2} \Phi_{1}(r, z)+O\left(F^{4}\right) \quad \text { and } \quad \zeta(r)=F^{2} \zeta_{1}(r)+O\left(F^{4}\right)
$$

and substitute into (2.1) to (2.4). To first order the problem is that $\Phi_{1}$ should satisfy Laplace's equation, and that the normal derivative at $z=-1$ and $z=0$ should be zero. At this order (2.4) does not appear. The solution for $\Phi_{1}$ is thus the flow from a point sink between two flat plates, and is given in [6]. Substituting this solution into (3.1) we may obtain $\zeta_{1}(r)$, so that a first approximation to the free surface shape is

$$
\begin{aligned}
\zeta(r)=F^{2} \zeta_{1}(r)+O\left(F^{4}\right) & =-\frac{1}{2} F^{2}\left(\Phi_{1 r}^{2}+\Phi_{1 z}^{2}\right)+O\left(F^{4}\right) \\
& =-\frac{F^{2}}{8 \pi^{2}}\left(H(r)^{2}+V(r)^{2}\right)+O\left(F^{4}\right)
\end{aligned}
$$

where $H(r)$ and $V(r)$ come from the derivatives of $\Phi_{1}$ evaluated on $z=0$, that is,

$$
H(r)=r A(r)+\sum_{\substack{k=-\infty \\ k \neq 0}}^{\infty} r B(r, k), \quad V(r)=A(r)+\sum_{\substack{k=-\infty \\ k \neq 0}}^{\infty}(1-2 k) B(r, k)
$$

where $A(r)=\left(r^{2}+1\right)^{-3 / 2}$ and $B(r, k)=\left(r^{2}+(1-2 k)^{2}\right)^{-3 / 2}$. This small Froude number solution converges to graphical accuracy with only 40 terms in each series. We will use it to compare with solutions to the following numerical scheme.

\section{Numerical method}

In this section we give a brief description of the numerical scheme employed to obtain an accurate solution to the system of equations (2.10)-(2.12).

The free surface was truncated some distance, $\sigma_{T}$, from the central point above the sink and discretised into $(N+1)$ intervals of length $\Delta \sigma$, that is, $\sigma_{1}=0, \sigma_{2}=$ $\Delta \sigma, \sigma_{3}=2 \Delta \sigma, \ldots, \sigma_{N+1}=N \Delta \sigma=\sigma_{\tau}$. Values of $\phi=\phi_{k}, k=1,2, \ldots, N$, and $\zeta_{k}, k=1,2, \ldots, N$, were assigned to each point. Using these values it is possible to compute $\zeta^{\prime}(\sigma)$ using finite differences, and then $r^{\prime}(\sigma)$ can be obtained using (2.10). Finally, $r(\sigma)$ can be obtained using numerical integration. The elliptic integrals can 
be obtained with an error of less than $10^{-8}$ using polynomial representations given in Abramowitz and Stegun [1].

The unknowns are thus $\phi_{k}, k=0,1,2, \ldots, N$, and $\zeta_{k}, k=0,1,2, \ldots, N$, and there are $2 N$ of these. The error at the midpoint of each interval in the integral equation can be evaluated, and the error in the Bernoulli condition at the endpoints of each interval provide $2 N-1$ equations. The final equation is simply that $\phi_{N}$ is extrapolated from the previous points. The evaluation of the integral is performed using a midpoint rule which therefore avoids the singularity as $\sigma \rightarrow s$. There is no point in using a higher order scheme for the integral because the error is dominated by the presence of this singularity, so higher order schemes give no greater accuracy. The error decays linearly for increasing $N$ for this integral. This system of $2 N$ equations is then solved using a Newton iteration scheme.

Careful analysis of the equation reveals another numerical problem. The coefficient of $\mathbf{E}$, the elliptic integral, in the integrand, while well-behaved, has a term of order $\Delta s^{2}$ in the denominator as $\sigma \rightarrow s$. The numerator of this term also has this behaviour, but numerically this means that the error in the numerator must be at most $O\left(\Delta s^{4}\right)$ or there will be an $O(1)$ error in this term. This was treated using a four-point interpolation formula and a higher order finite difference scheme for all interpolation and differentiation. This was found to give an accurate representation of this term.

The iterations were found to converge very well, and solutions were computed over a range of values of $F$. The actual free surface shapes, however, were found to converge rather slowly, and $N=400$ and $\Delta s=0.025$ were required along the free surface to obtain solutions to graphical accuracy.

\section{Results and discussion}

Figure 3 shows a comparison of the numerically computed free surface shape with (a) the small Froude number solution at $F=0.5$, (b) the small Froude number solution and the solution of Forbes et al. [6] at $F=1.25$. The fundamental singularities method, [6], failed to converge for $F>1.5$. Similarly, it was found that a method using evaluation of the error at the endpoints of the discrete intervals (rather than the midpoints), but using the formulation given in this paper, broke down at about the same value of $F$. The agreement of the numerical scheme with the small Froude number solution is very good at $F=0.5$, but the deepest point of the dip is slightly different to the full nonlinear solution at $F=1.25$. Agreement with the solution of [6] is excellent.

Figure 4 shows the development of a ring of very small waves on the free surface as $F$ increases. The maximum $F$ was found to be at around 3.24, when the innermost wave rose up to form a stagnation ring around the central stagnation point, much as happened in the case of infinite depth. An analysis of the nature of this stagnation line 


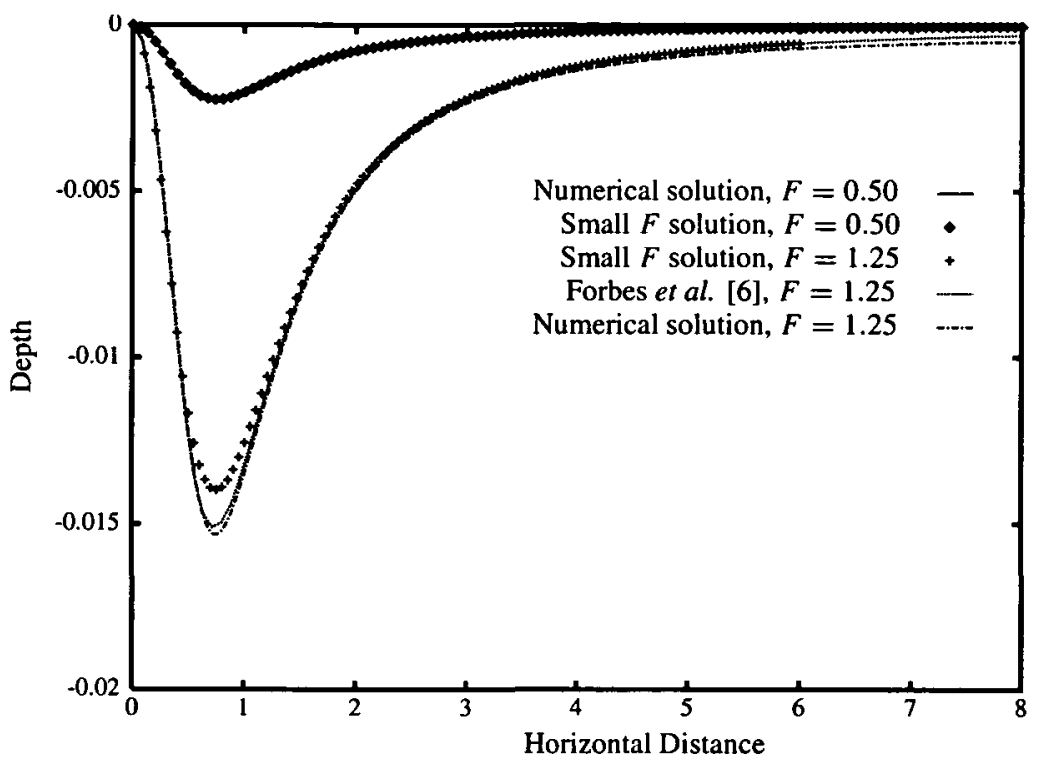

FIGURE 3. Comparison of solutions using the small Froude number expansion with numerical solution in this paper for $F=0.5$, and small $F$ solution with the fundamental singularities method of Forbes $e t$ $a l$. [6] and the numerical method of this paper for $F=1.25$.

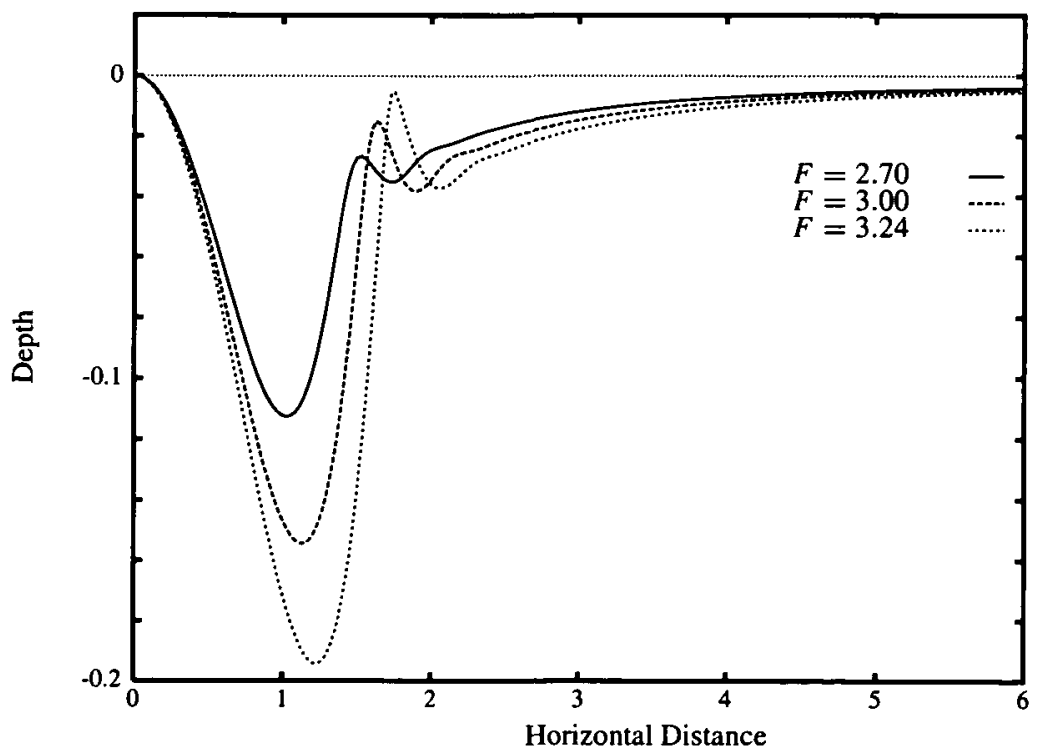

FIGURE 4. Axisymmetric free surface shapes for increasing values of $F$ up to that near the limiting value, $F=2.7,3.0,3.24$. 
is given in [17].

These results are in qualitative agreement with the behaviour of flow into a point sink in a fluid of infinite depth. However, it is clear that the numerical schemes to solve this problem are variable in their success. The "fundamental singularities" method described in [6], and variations on the scheme used in this paper were unsuccessful in computing solutions beyond $F=1.5$. In fact if the singularity is removed as was done in Forbes and Hocking [4], the Newton method failed to converge for any value of $F$ even when the starting guess was extremely good.

In terms of the general result, it is of interest to compare it to various experimental, analytical and numerical work on this problem. In particular, it is of interest to examine the critical drawdown Froude number, $F=F_{C}$ and how this relates to the value of $F$ at which the steady solution computed here fails, that is, when the stagnation ring forms. Are the two events related in some way? Craya [3] performed an analysis which suggested that the critical drawdown Froude number would be around $F_{C}=2.54$ for the case of a point sink on the bottom of the fluid region, and Wood [18] suggested a modification for a fluid of infinite depth which gives $F \approx 5.08$ (since the flux is doubled). Experiments by Gariel [7], $F_{C} \approx 2.55$, Harleman et al. [8], $F_{C} \approx 1.61$, Lubin and Springer [13], $F_{C} \approx 2.53$, and Lawrence and Imberger [12], $0.3<F_{C}<5.3$, all obtained critical values around these expected values. Numerical experiments $[2,19]$ computing the dip formed in a free surface in a circular tank with a hole in the bottom obtained a critical value of $F_{C} \approx 3$. Jirka and Katavola [11] obtained much lower values, but were using layers separated by a very diffuse interface. Forbes and Hocking [5] obtained solutions for flow including circulation about a withdrawal "bath" hole, but taking the minimum Froude number at which they obtained solutions with an air cone through the middle gives a critical value of $F=\sqrt{2} \pi \approx 4.4$, a little higher than the other values, but for a slightly different situation (a particular outflow velocity profile was assumed across the outlet hole).

The values at which the stagnation ring forms in both this case of the sink on the bottom, and of the sink in infinite depth, [4], $F_{C} \approx 6.4$, agree quite well with the value obtained using the approximate analyses of Craya [3] and Wood [18]. This quite strongly suggests that the formation of this stagnation ring is the physical event which triggers the collapse of the single layer flow, or if there is another layer above of different density, the transition to a flow in which both layers are entering the sink.

It would seem likely that the formation of the secondary stagnation ring is the prelude to some kind of wave breaking event, and at higher Froude number, an unsteady flow situation would occur. In other words, either there is an unsteady flow or, if there is a layer of different density above, there may also be a steady flow in which fluids from both layers are entering the sink. This would be analogous to the two dimensional work of Hocking [9], for example. Work is currently under way to determine if either or both of these flow types is possible. 


\section{References}

[1] M. Abramowitz and I. A. Stegun (eds.), Handbook of Mathematical Functions (Dover, New York, 1970).

[2] J. H. Baek and H. Y. Chung, "Numerical analysis on axisymmetric draining from a cylindrical tank with a free surface", J. Comp. Fluid Dynamics 6 (1998) 413-425.

[3] A. Craya, "Theoretical research on the flow of nonhomogeneous fluids", La Houille Blanche 4 (1949) 44-55.

[4] L. K. Forbes and G. C. Hocking, "Flow caused by a point sink in a fluid having a free surface", J. Austral. Math. Soc. Ser. B 32 (1990) 231-249.

[5] L. K. Forbes and G. C. Hocking, “The bath-plug vortex", J. Fluid Mech. 284 (1995) 43-62.

[6] L. K. Forbes, G. C. Hocking and G. A. Chandler, "A note on withdrawal through a point sink in fluid of finite depth", J. Austral. Math. Soc. Ser. B 37 (1996) 406-416.

[7] P. Gariel, "Experimental research on the flow of nonhomogeneous fluids", La Houille Blanche 4 (1949) 56-65.

[8] D. R. F. Harleman, R. L. Morgan and R. A. Purple, "Selective withdrawal from a vertically stratified fluid", in Proc. Int. Assoc. Hyd. Res., 8th Congress, Montreal (1959).

[9] G. C. Hocking, "Supercritical withdrawal from a two-layer fluid through a line sink", J. Fluid Mech. 297 (1995) 37-47.

[10] J. Imberger and P. F. Hamblin, "Dynamics of lakes, reservoirs and cooling ponds", Ann. Rev. Fluid Mech. 14 (1982) 153-187.

[11] G. H. Jirka and D. S. Katavola, "Supercritical withdrawal from two-layered fluid systems. Part 2-Three dimensional flow into a round intake", J. Hyd. Res. 17 (1979) 53-62.

[12] G. A. Lawrence and J. Imberger, "Selective withdrawal through a point sink in a continuously stratified fluid with a pycnocline", Technical Report ED-79-002, Dept of Civil Eng., University of Western Australia, Australia, 1979.

[13] B. T. Lubin and G. S. Springer, "The formation of a dip on the surface of a liquid draining from a tank", J. Fluid Mech. 29 (1967) 385-390.

[14] C. Sautreaux, "Mouvement d'un liquide parfait soumis à la pesanteur. De termination des lignes de Courant", J. Math. Pures Appl. 7 (1901) 125-159.

[15] E. O. Tuck and J.-M. Vanden-Broeck, "A cusp-like free-surface flow due to a submerged source or sink", J. Aust. Math Soc. Ser. B 25 (1984) 443-450.

[16] J.-M. Vanden-Broeck and J. B. Keller, "Free surface flow due to a sink", J. Fluid Mech. 175 (1987) 109-117.

[17] J.-M. Vanden-Broeck and J. B. Keller, "An axisymmetric free surface with a $120^{\circ}$ along a circle", J. Fluid Mech. 342 (1997) 403-409.

[18] I. R. Wood, "Selective withdrawal from two-layer fluid", J. Hyd. Div. (ASCE) HY12 (1978) 1647-1659.

[19] Q. Zhou and W. P. Graebel, "Axisymmetric draining of a cylindrical tank with a free surface", J. Fluid Mech. 221 (1990) 511-532. 